\title{
DESIGN OF METAMATERIAL INSPIRED HIGH GAIN PATCH ANTENNA
}

\author{
T. Sathiyapriya, V. Gurunathan and A. Shafeek \\ Department of Electronics and Communication Engineering, Dr. Mahalingam College of Engineering and Technology, India
}

\begin{abstract}
This paper investigates the single band microstrip antenna loaded with meta-material. The main objective is to enhance the gain and directivity of the conventional microstrip patch antenna operating at $X$ band. The Square SRR is acting as a metamaterial that is designed to exhibit negative properties at $10 \mathrm{GHz}$. The parameters of unit cell metamaterial structure, simulated in CST Microwave Studio 2015, are extracted from S-parameters using Nicholson-Ross-Weir method. A unit cell SRR is iterated in various configurations and employed as an intermediate layer between patch and ground. By inserting a $6 \times 4$ array of metamaterial below the patch, it achieves Gain and directivity of 7.9dBi with $100 \%$ efficiency. The antenna resonates at $11.8 \mathrm{GHz}$ with $-24 \mathrm{~dB}$ reflection coefficient and also has good radiation characteristic. Being a high gain antenna resonates at $X$ band, it can be used for satellite applications.
\end{abstract}

\section{Keywords:}

Metamaterial, Split Ring Resonators, X Band RADAR, Advanced Design System (ADS)

\section{INTRODUCTION}

In wireless communication technology and communication systems, a microstrip patch antenna is widely used due to their advantages such as low profile, low cost, simple design and easy to manufacture. The metallic patch is normally made with a metal such as copper, gold and nickel. The feeding methods of the microstrip patch antenna can be classified into two categories, contacting and non-contacting. The RF power is fed directly to the microstrip line in the contacting method, whereas in noncontacting electromagnetic feed coupling is done to transfer patch between the microstrip line and radiating patch [2]. At the same time, it has various disadvantages such as narrow bandwidth, low gain, and low directivity. But for narrowband applications like Satellite communication, antenna should possess high gain and directivity. To increase gain, literature reported techniques are loading of an antenna in different aspects like Electromagnetic Band Gap (EBG) structures, Defected Ground Structures (DGS), highly-reflective surfaces (HRS), Artificial Magnetic Conductors (AMC) and Metamaterials [17].

This paper focuses on adding Metamaterial with conventional microstrip antenna that significantly improves gain and directivity at X band. Metamaterials are artificial materials and it illustrates the properties that are not found in nature. In 1968, Veselago theoretically showed that it exhibits different properties such as negative permittivity and negative permeability compared to ordinary materials [3]. In 1999, Pendry proposes a concept of Split Ring Resonator (SRR) that will be an equivalent of the metamaterial. It is formed by two rings with a split on opposite sides, behaves as an LC resonator that can be excited by a time-varying external magnetic field component in the normal direction of a resonator. The gaps between the rings produce capacitance value, it leads to low radiation losses and high- quality factor [4]. In 2013, Mandal et al. presented a high gain wideband. U-shaped patch antenna with two equal arms on Poly Tetra Fluoro Ethylene (PTFE) substrate. An inverted U-shaped slot is introduced on the circular or square shaped ground plane just under the U-shaped patch. Maximum impedance bandwidth of $86.79 \%(4.5 \mathrm{GHz}-11.4 \mathrm{GHz})$ is obtained with a circular shaped ground plane with diameter $36 \mathrm{~mm}$. The proposed antenna is simple in structure compared to the regular stacked or coplanar parasitic patch antenna with a gain of $4.1 \mathrm{dBi}$ and is suitable for wireless communications [7]. Attia et al. (2009) proposed a novel engineered magnetic superstrate to enhance the gain and efficiency of a microstrip patch antenna. The patch antenna with magnetic superstrate resonating at $2.2 \mathrm{GHz}$, results in $3.4 \mathrm{~dB}$ gain enhancement and $17 \%$ efficiency enhancement. These improvements were achieved while having an insignificant effect on the antenna bandwidth [9]. In 2003, Jieh designed a single fed circularly polarized equilateral triangular microstrip antenna with enhanced antenna gain. The circular polarization design is obtained by placing three triangular slots below the radiating patch. The antenna gain has been increased by $3.3 \mathrm{dBi}$ compared to a conventional patch antenna. The proposed antenna is fabricated for experimental evaluation [10]. In 2012, Yingli et al. proposed a metamaterial unit cell with low refractive index and it is optimized to enhance the gain of the patch antenna and beam focusing ability. The effective material parameters of the unit cell are extracted, and the unit cell forms a planar three-layer metamaterial structure used as a superstrate for gain enhancement of a patch antenna at $10 \mathrm{GHz}$ has a $7.8 \mathrm{dBi}$. The measurements have shown that the effective refractive index of the Metamaterial under normal incidence is close to zero over a frequency range from $9.45 \mathrm{GHz}$ to $10.7 \mathrm{GHz}$ [8]. In 2018, Saravanan et al. investigated a phi-shaped metamaterial superstrate with the square patch antenna. The antenna achieves a gain of $7.94 \mathrm{~dB}$ at its resonance frequency $2.4 \mathrm{GHz}$. Phi shaped slot is etched on a square conductor is considered as a unit cell and it is validated [18].

In this paper, we consider the problem of enhancing the gain and directivity of an inset feed microstrip patch antenna using Split Ring Resonator based metamaterial. The metamaterial is inserted as an intermediate layer between patch and ground plane. The edge-coupled SRR (EC-SRR) consists of two copper rings and one metal layer at the ground plane is used. The other structures of SRR are Broadside Couple Split ring resonator (BCSRR) [12], non bi-anisotropic Split ring resonator (NB-SRR) [13], Spiral Split Ring Resonator (S-SRR), Open Split Ring Resonator (O-SRR), H-shaped Split Ring Resonator (HS-SRR), Quadruple P shaped Split Ring Resonator (QPS-SRR).

The paper is organized as follows: section 2 explains the design procedure of a microstrip patch antenna loaded with Metamaterial. Section 3 is about the simulation results achieved by employing Split Ring Resonator. Section 4 gives the conclusion of the proposed work. 


\section{ANTENNA DESIGN}

\subsection{CONVENTIONAL RECTANGULAR PATCH ANTENNA}

Microstrip patch antenna consists of a radiating patch on one side backed by a ground plane. The conventional rectangular patch antenna is designed on Roger RT-Duroid 5880 substrate for $10 \mathrm{GHz}$ with the dimensions of $10 \times 17 \mathrm{~mm}$ and simulated in ADS software. The dielectric constant $\left(\varepsilon_{r}\right)$ of the substrate is 2.2 and dielectric loss tangent $(\delta)$ is $0.0009 \mathrm{~mm}$ as per the substrate specifications. The Fig.1 depicts the schematic diagram of the conventional patch antenna. The width $(W)$ and length $(L)$ of the patch is calculated using Eq.(1) and Eq.(2) [14],

$$
\begin{gathered}
W=\frac{c}{2 f_{r}} \sqrt{\frac{2}{\varepsilon_{r}+1}} \\
L=\frac{c}{2 f_{r} \sqrt{\varepsilon_{\text {reff }}}}-2 \Delta L
\end{gathered}
$$

where, $W$ is the width of the patch, $\mathrm{c}$ is the speed of light, $\varepsilon_{r}$ is dielectric constant and $\varepsilon_{\text {reff }}$ is Effective dielectric constant.

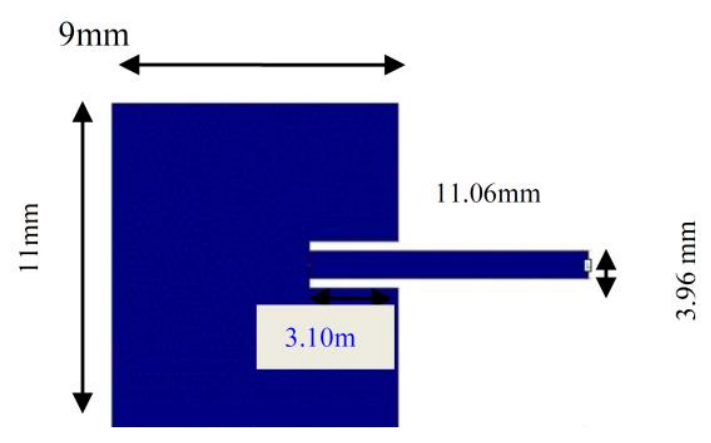

Fig.1. Conventional Rectangular Patch Antenna

Table.1. Dimensions of the patch antenna

\begin{tabular}{|c|c|}
\hline Part & Dimension $(\mathbf{m m})$ \\
\hline Patch width & 11 \\
\hline Patch length & 9 \\
\hline Feed line width & 3.9663 \\
\hline Feed line depth & 11.0613 \\
\hline Copper thickness & 0.035 \\
\hline Substrate thickness & 1.585 \\
\hline
\end{tabular}

\subsection{SPLIT RING RESONATOR}

The Fig.2 illustrates the unit cell of square SRR. To consider it as metamaterial double negative property is verified from parametric values such as of permittivity $(\varepsilon)$, permeability $(\mu)$ and refractive index $(n)$ [14].

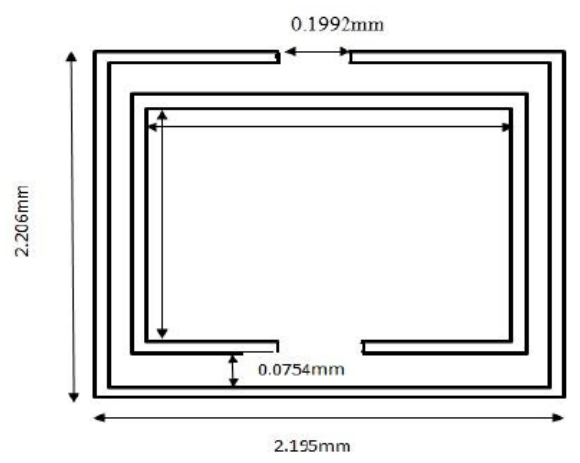

Fig.2. Schematic Diagram of SRR

The formula for calculating the value of permittivity and permeability [15] are given in Eq.(3) and Eq.(4).

$$
\begin{gathered}
\mu_{r}=\frac{2 c\left(1-v_{1}\right)}{\omega d i\left(1-v_{2}\right)} \\
\varepsilon_{r}=\mu_{r}+\frac{2 s_{11} c i}{\omega d} \\
V_{1}=s_{11}+s_{21} \\
V_{2}=s_{21}-s_{11}
\end{gathered}
$$

where, ' $\omega$ ' is Frequency in Radian, ' $d$ ' is the Thickness of the substrate, ' $c$ ' is the speed of light, $V_{1}$ is voltage maxima and $V_{2}$ is voltage minima.

Table.2. Dimensions of Split Ring Resonator

\begin{tabular}{|c|c|}
\hline Parts & Dimension $(\mathbf{m m})$ \\
\hline Outer Ring width & 2.201 \\
\hline Outer Ring length & 2.206 \\
\hline Inner ring width & 1.848 \\
\hline Inner Ring length & 1.843 \\
\hline Gap & 0.1992 \\
\hline
\end{tabular}

The square SRR has been analyzed by using CST simulation tool as per the specifications mentioned in the table. In order to calculate the $S$ parameters, the unit cell is placed between two waveguide ports. Y plane is defined as Perfect Electric Boundary (PEB) and the $\mathrm{Z}$ plane is defined as Perfect Magnetic Boundary (PMB). The SRR design is shown in Fig.3, to verify the double negative property of the unit cell MATLAB R2013b simulation tool is used to extract the values of $\mathrm{S}$ parameters.

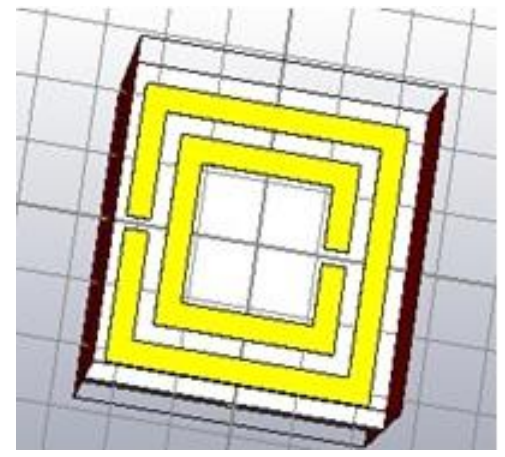

Fig.3. SRR design 


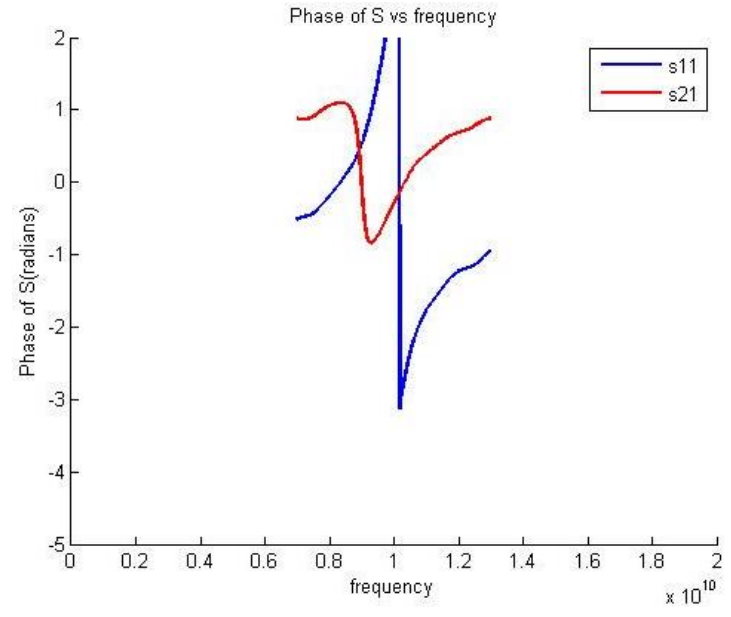

Fig.4. S Parameters of metamaterial unit cell

The Fig. 4 shows that $S_{11}$ and $S_{21}$ parameters have phase reversal at frequency of $10 \mathrm{GHz}$. Hence the designed unit cell SRR is working as a metamaterial at its resonance frequency.

\subsection{PROPOSED ANTENNA DESIGN WITH SRR}

The SRR array is placed as a key building element between the patch and the ground plane which resonates at a frequency of $10 \mathrm{GHz}$. The first requirement of the SRR array is the optimum spacing between the elements of the array. The performance of an array depends on the number of rings, pattern of arrangement and orientation of the rings. The unit cell SRR presented in section II is employed to design the Split Ring Resonator array and it can produce significant gain and directivity enhancement. The proposed antenna is designed on Roger RT-duroid 5880 substrate of thickness $=1.585 \mathrm{~mm}$, dielectric constant $\left(\varepsilon_{r}\right)=2.2$ and loss tangent is 0.0009 . Each square unit cell has an area of $4.84 \mathrm{~mm}^{2}$. The SRR unit cells are separated by different width and length from each other according to their inter-element spacing. The various SRR array designs are presented in Fig.5-Fig.8. Advanced Design System (ADS) 2016 software tool is used to design and simulate proposed antenna.

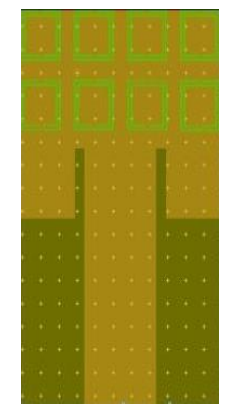

Fig.5. Proposed Antenna with various iterations of SRR array $2 \times 4$

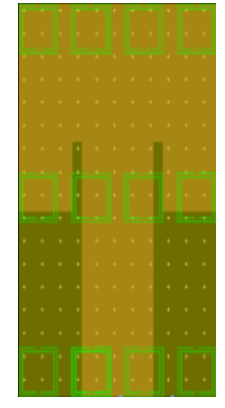

Fig.6. Proposed Antenna with various iterations of SRR array $3 \times 4$ SRR

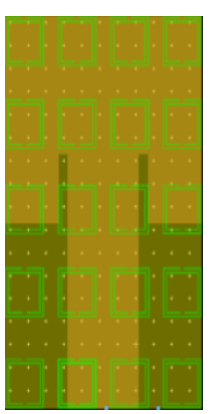

Fig.7. Proposed Antenna with various iterations of SRR array $5 \times 4$

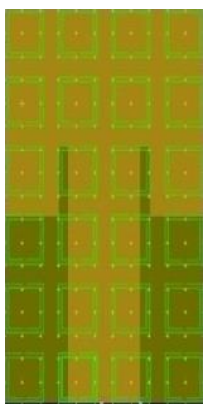

Fig.8. Proposed Antenna with various iterations of SRR array $6 \times 4$

\section{RESULTS AND DISCUSSIONS}

The reflection characteristics of the various antenna designs are indicated in Fig.9-Fig.10. The return loss should be minimized as much as possible for better performance at the resonance frequency. Antenna with $6 \times 4$ unit cell array resonates with suitable return loss at $11.86 \mathrm{GHz}$. It achieves an impedance bandwidth of $350 \mathrm{MHz}$ ranging from $11.71 \mathrm{GHz}$ to $12.06 \mathrm{GHz}$. The Fig. 8 shows the return loss of $-24.407 \mathrm{~dB}$ is obtained at a resonant frequency of $11.86 \mathrm{GHz}$.

Various antenna parameters are observed to evaluate the performance of an Antenna. The efficiency of the proposed antenna is far better than the efficiency of a conventional rectangular patch antenna. The efficiency of the $6 \times 4$ array antenna is $100 \%$ while the efficiency of a single patch antenna was $98 \%$. By increasing the number of unit cells in the rectangular patch antenna, the antenna parameters such as Efficiency has been enhanced. 


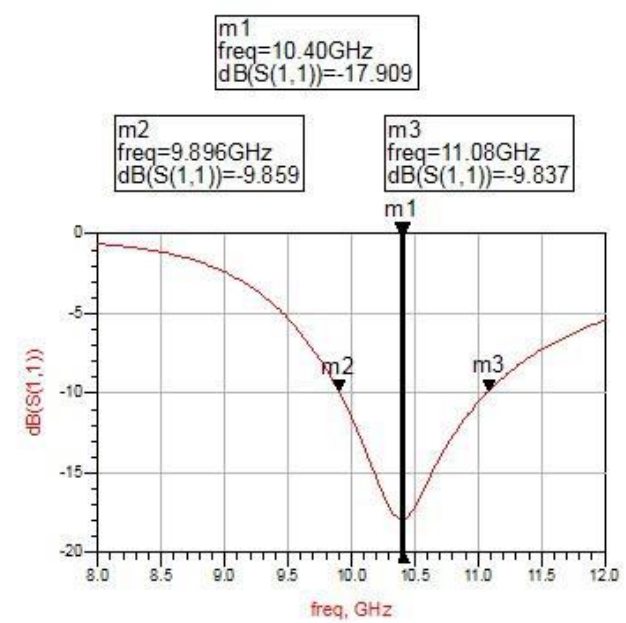

Fig.9. Simulated Return Loss of $2 \times 4$ array

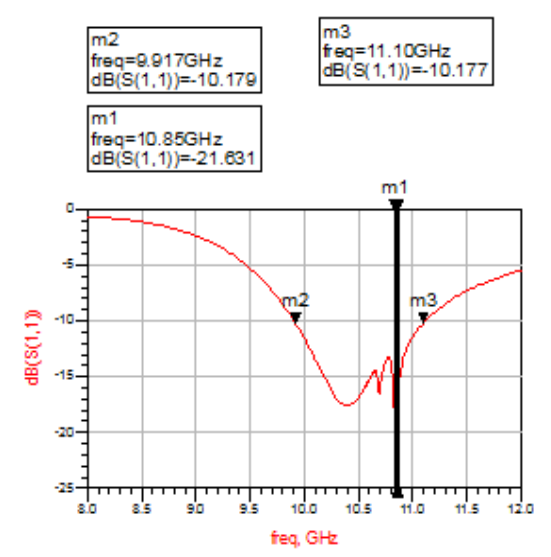

Fig.10. Simulated Return Loss of $3 \times 4$ array

The various antenna structure and its parameters such as Gain, Directivity, Efficiency, Bandwidth has been compared and is depicted in the Table.3. From the table, it is observed that the gain $(7.9 \mathrm{dBi})$, directivity $(7.9 \mathrm{dBi})$ and efficiency $(99.714 \%)$ of $6 \times 4$ SRR array is better compared to other designed antenna structure because of increasing the number of unit cell (SRR) in the antenna. It is concluded that the $6 \times 4$ array structure is better in terms of gain and directivity whereas a $2 \times 4$ array structure is better in terms of improved impedance bandwidth.

Table.3. Comparison of various Configurations

\begin{tabular}{|c|c|c|c|c|}
\hline Parameters & $\mathbf{2 \times 4}$ & $\mathbf{3 \times 4}$ & $\mathbf{5 \times 4}$ & $\mathbf{6 \times 4}$ \\
\hline $\begin{array}{l}\text { Layout } \\
\text { Design }\end{array}$ & & & & \\
& & & & \\
\hline Return loss (dB) & -17.909 & -21.631 & -17.550 & -21.407 \\
\hline Gain $(\mathrm{dBi})$ & 6.9 & 7.321 & 7.444 & 7.92563 \\
\hline Directivity $(\mathrm{dBi})$ & 6.9 & 7.527 & 7.444 & 7.9233 \\
\hline Bandwidth $(\mathrm{GHz})$ & 1.24 & 1.19 & 0.78 & 0.35 \\
\hline Efficiency $(\%)$ & 100 & 95.357 & 99.649 & 99.714 \\
\hline
\end{tabular}

\section{CONCLUSION}

In this investigation, a microstrip patch antenna loaded with metamaterial has been designed and its performance is analyzed. Double negative property of the metamaterial is proved by extracting the values of permittivity and permeability from $S$ parameters. It is shown that inclusion of $6 \times 4$ SRR array produces the gain of $7.925 \mathrm{dBi}$ and directivity of $7.9233 \mathrm{dBi}$. It is observed that patch with $2 \times 4$ arrays gives a wide bandwidth of $1.24 \mathrm{GHz}$ with a low gain of $6.9 \mathrm{dBi}$. Hence for narrow band applications antenna with $6 \times 4$ SRR provides large gain and directivity. The proposed antenna is designed with Roger RT Duroid 5880 Substrate. It achieves a radiation efficiency of $99 \%$ and it is suitable for satellite applications.

\section{REFERENCES}

[1] Y. Lo, D. Solomon and W. Richards, "Theory and Experiment on Microstrip Antennas", IEEE Transactions on Antennas and Propagation, Vol. 27, No. 2, pp. 137-145, 1979.

[2] Ramesh Garg, "Progress in Microstrip Antenna", IETE Technical Review, Vol. 18, No. 2-3, pp. 2-3, 2015.

[3] V.G. Veselago, "The Electrodynamics of Substances with Simultaneously Negative Values of $\varepsilon$ and $\mu$ ", Soviet Physics Uspekhi, Vol. 10, No. 4, pp. 509-514, 1968.

[4] John B. Pendry and David R. Smith, "Reversing Light: Negative Refraction", Physics Today, Vol. 57, No. 6, pp. 3137, 2003.

[5] Nader Engheta and Richard W. Ziolkowski, "Electromagnetic Metamaterials, Physics, and Engineering Explorations", Wiley IEEE Press, 2006.

[6] Richard W. Ziolkowski, "Double Negative Metamaterial Design, Experiments and Application", IEEE Transactions on Microwave Theory and Techniques, Vol. 51, No. 7, pp. 233-247, 2003.

[7] Kaushik Mandal and Partha Prtim Sarkar, "High Gain Wideband U-shaped Patch Antennas with Modified Ground Planes", IEEE Transactions on Antennas and Propagation, Vol. 61, No. 4, pp. 2279-2282, 2013

[8] Dongying Li, Zsolt Szabo, Xianming Qing, Er Ping Li and Zhi Ning Chen, "A High Gain Antenna with an Optimised Metamaterial Inspired Superstrate", IEEE Transactions on Antennas and Propagation, Vol. 60, No. 12, pp. 6018-6023, 2012.

[9] H. Attia, L. Yousefi, M.M. Bait Suwailam, M.S. Boybay and O.M. Ramahi, "Enhanced Gain Microstrip Antenna using Engineered Superstrates", IEEE Antennas and Wireless Propagation Letters, Vol. 8, pp. 1198-1201, 2009.

[10] Jieh-Sen Kuo and Gui-Bin Hsieh, "Gain Enhancement of a Circularly Polarised Equilateral Triangular Microstrip Antenna with a Slotted Ground Plane", IEEE Transactions on Antennas and Propagation, Vol. 51, No. 7, pp. 16521656, 2003.

[11] J. Huang, "The Finite Ground Plane Effect on the Microstrip Antenna Radiation Patterns", IEEE Transactions on Antennas and Propagation, Vol. 31, No. 4, pp. 649-653, 1983.

[12] R. Marques, F. Mesa, J. Martel and F. Medina, "Comparative Analysis of Edge and Broadband Coupled 
Split Ring Resonator for Metamaterial Design Theory and Experiments", IEEE Transactions on Antennas and Propagation, Vol. 51, No. 10, pp. 2572-2580, 2003

[13] R. Marques, R. Medina and, E.I. Raffi, "Role of Bianisotropy in Negative Permeability and Left-handed Metamaterials", Physics Review B, Vol. 65, pp. 144440144448, 2002.

[14] R.A. Shelby, D.R. Smith and S. Schultz, "Experimental Verification of a Negative Index of Refraction", Science, Vol. 292, No. 5514, pp. 77-79, 2001.

[15] D.R. Smith, S. Schultz, P. Markos and C.M. Soukoulis, "Determination of Effective Permittivity and Permeability of Metamaterials from Reflection and Transmission Coefficients", Physical Review B, Vol. 65, pp. 195104195105, 2002.

[16] J.G. Josh, Shyam S. Pattnaik, Swapna Devi and M.R. Lohokare, "Electrically Small Patch Antenna Loaded with
Metamaterial", IETE Journal of Research, Vol. 56, No. 6, pp. 373-379, 2010

[17] Raoul O. Ouedraogo, Edward J. Rothwell, Alejandro R. Diaz, Kazuko Fuchi and Andrew Temme, "Miniaturization of Patch Antenna using a Metamaterial Inspired Technique", IEEE Transactions on Antennas and Propagation, Vol. 60, No. 5, pp. 2175-2182, 2012

[18] Chirag Arora, Shyam S. Pattnaik and R.N. Baral, "Performance Enhancement of Patch Antenna Array for 5.8 $\mathrm{GHz}$ Wi-MAX applications using Metamaterial Inspired Technique", International Journal of Electronics and Communication, Vol. 79, pp. 124-131, 2017.

[19] M. Saravanan, V. Berlin Geo and S.M. Umarani, "Gain Enhancement of Patch Antenna Integrated with Metamaterial Inspired Superstrate", Journal of Electrical Systems and Information Technology, Vol. 5, No. 3, pp. 263270,2018 